\title{
Low-Noise Amplification and Frequency Conversion with a Multiport Microwave Optomechanical Device
}

\author{
C. F. Ockeloen-Korppi, ${ }^{1}$ E. Damskägg, ${ }^{1}$ J.-M. Pirkkalainen, ${ }^{1}$ T. T. Heikkilä, ${ }^{2}$ F. Massel, ${ }^{2}$ and M. A. Sillanpää ${ }^{1,{ }^{*}}$ \\ ${ }^{1}$ Department of Applied Physics, Aalto University, P.O. Box 11100, FI-00076 Aalto, Finland \\ ${ }^{2}$ Department of Physics, Nanoscience Center, University of Jyvaskyla, \\ P.O. Box 35 (YFL), FI-40014 University of Jyvaskyla, Finland
}

(Received 20 June 2016; revised manuscript received 22 September 2016; published 28 October 2016)

\begin{abstract}
High-gain amplifiers of electromagnetic signals operating near the quantum limit are crucial for quantum information systems and ultrasensitive quantum measurements. However, the existing techniques have a limited gain-bandwidth product and only operate with weak input signals. Here, we demonstrate a two-port optomechanical scheme for amplification and routing of microwave signals, a system that simultaneously performs high-gain amplification and frequency conversion in the quantum regime. Our amplifier, implemented in a two-cavity microwave optomechanical device, shows $41 \mathrm{~dB}$ of gain and has a high dynamic range, handling input signals up to $10^{13}$ photons per second, 3 orders of magnitude more than corresponding Josephson parametric amplifiers. We show that although the active medium, the mechanical resonator, is at a high temperature far from the quantum limit, only 4.6 quanta of noise is added to the input signal. Our method can be readily applied to a wide variety of optomechanical systems, including hybrid optical-microwave systems, creating a universal hub for signals at the quantum level.
\end{abstract}

DOI: 10.1103/PhysRevX.6.041024

Subject Areas: Quantum Physics

Recent advances in near-quantum-limited amplifiers in the microwave-frequency regime have led to breakthroughs in the understanding of quantum measurement processes [1-4] and are necessary in quantum error correction and feedback [5-7]. Of particular interest for most applications are phase-insensitive linear amplifiers, which provide a faithful reconstruction of both quadratures of the input. Such amplifiers are bound by the standard quantum limit (SQL), which states that at high gain at least half an energy quantum of noise is added to the input signal [8]. This limit has been approached with Josephson parametric amplifiers [9-14]; however, such amplifiers are limited to relatively weak input signals. Optomechanical amplifiers or detectors, utilizing the interaction between electromagnetic waves and a mechanical resonator inside a cavity [15], have been demonstrated in the microwave and optical regime [16-19], but existing techniques suffer from a limited gain and bandwidth as well as noise levels well above the SQL.

In this paper, we demonstrate a two-port optomechanical device, motivated by the proposal in Ref. [20]. It consists of two electromagnetic cavities with different resonant frequencies, simultaneously coupled [21-23] to a single mechanical resonator. In the presence of appropriately

*mika.sillanpaa@aalto.fi

Published by the American Physical Society under the terms of the Creative Commons Attribution 3.0 License. Further distribution of this work must maintain attribution to the author(s) and the published article's title, journal citation, and DOI. chosen external pump tones, the mechanical resonator mediates interaction between the cavities, enabling strong amplification of a signal reflected from one of the cavities. Moreover, the scheme supports frequency-converting amplification, where a signal incident in one cavity can irradiate out from the other cavity at a completely different frequency, while being amplified at the same time. As we show theoretically, our scheme can reach the SQL for both of these processes. Unlike existing optomechanical amplifiers $[16,17]$, the bandwidth of amplification in our scheme can be in principle increased up to the cavity linewidth, and the product of gain and bandwidth has no fundamental limit. Remarkably, we show in experiment that the quantum limit can be closely approached even at a high temperature where the mode occupation numbers $\gg 1$, which allows for an interpretation in terms of reservoir engineering [20,24-27].

In a microwave-frequency optomechanical [28] experiment, we obtain a gain of $41 \mathrm{~dB}$ with a gain-bandwidth product of $137 \mathrm{kHz}$, while adding only 4 quanta of noise above the SQL. Additionally, we show how the multimode system can act as a spectrally pure microwave source in the regime of self-oscillations. Finally, using an alternate pump scheme, our system also supports frequency conversion without amplification, similar to previous experiments in a range of systems $[22,23,29,30]$. With that method, we observe near-unity conversion efficiency, wide bandwidth, and added noise on the single photon level.

Our setup is shown in Fig. 1 and discussed in detail in the Supplemental Material [31]. At the heart of our device is a suspended aluminium drum resonator [3], with a resonant frequency of $\omega_{m}=2 \pi \times 8.3 \mathrm{MHz}$ and a linewidth of 

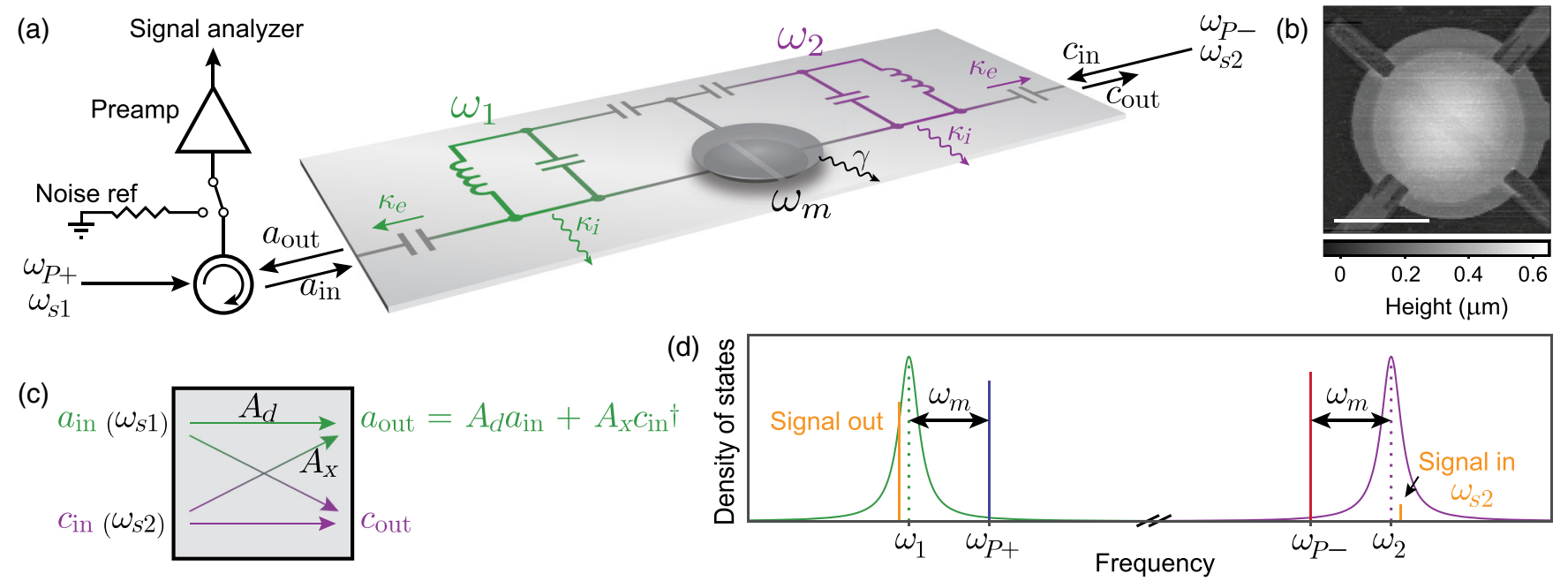

FIG. 1. Experimental setup. (a) Schematic of our device, showing two $L C$ microwave cavities $\left(\omega_{1}, \omega_{2}\right)$ both coupled to a central mechanical drum resonator $\left(\omega_{m}\right)$ as well as an individual feed line. Signals $\left(\omega_{s 1}, \omega_{s 2}\right)$ and pumps $\left(\omega_{P+}, \omega_{P-}\right)$ are fed to the cavities as shown. The output of cavity 1 is preamplified and measured with a signal analyzer. (b) Atomic force micrograph of the drum resonator. Scale bar is $10 \mu \mathrm{m}$ long. (c) Conceptual two-port amplifier, exhibiting both direct $\left(A_{d}\right)$ and cross $\left(A_{x}\right)$ gain. (d) Schematic representation of the cavity modes and pump frequencies used to realize a two-port amplifier. As an example, an input signal with frequency $\omega_{s 2}$ is injected to cavity 2 , with the frequency-converted and amplified output emerging from cavity 1.

$\gamma=2 \pi \times 100 \mathrm{~Hz}$, fabricated on a quartz substrate. The drum is surrounded by two inductor-capacitor $(L C)$ cavities, with resonant frequencies $\omega_{1}=2 \pi \times 7.0 \mathrm{GHz}$ and $\omega_{2}=2 \pi \times 8.4 \mathrm{GHz}$, respectively. The mechanical resonator is suspended over two electrodes to form a variable capacitance in each cavity, simultaneously coupling the mechanical motion to both cavities. Each cavity is capacitively coupled to an individual transmission line with a strong coupling rate, $\kappa_{e}=2 \pi \times 4.8 \mathrm{MHz}$, compared to the internal loss rate, $\kappa_{i}=2 \pi \times 0.50 \mathrm{MHz}$. The total cavity linewidth is $\kappa=\kappa_{i}+\kappa_{e}$.

The two-mode amplifier is created by injecting two pump tones [Fig. 1(d)], one at the blue mechanical sideband of cavity $1\left(\omega_{P+}=\omega_{1}+\omega_{m}\right)$, and the other at the red sideband of cavity $2\left(\omega_{P-}=\omega_{2}-\omega_{m}\right)$. In the presence of strong pump tones, the optomechanical interaction is effectively linear and enhanced by the cavity, with coupling strengths $G_{+}=\sqrt{n_{1}} g_{1}$ and $G_{-}=\sqrt{n_{2}} g_{2}$, where $g_{i}$ is the single-photon coupling strength and $n_{i}$ the photon occupation of cavity $i$. We also define $\mathcal{G}^{2}=\left(G_{-}^{2}-G_{+}^{2}\right)$ and the effective damping $\gamma_{\text {eff }}=\gamma+4 \mathcal{G}^{2} / \kappa$ of the mechanical resonator. Quantum-limited amplification in related multicavity configurations have been theoretically proposed $[20,32,33]$. Reference [20] treats a special case of equal pump tones, $G_{-}=G_{+}$, whereas Ref. [32] discusses an inverted dissipation hierarchy, where the linewidth of the mechanical resonator is much larger than $\kappa$. The latter case, using cavity damping to enhance $\gamma$, was experimentally realized in a work simultaneous to ours [34]. In our work, we treat the case $G_{-} \gtrsim G_{+}$and $\gamma_{\text {eff }} \ll \kappa$. Moreover, we introduce the possibility for frequency-converting amplification, in addition to unity-gain frequency conversion $[22,23,29,30]$.

The effective Hamiltonian describing the coupling between the subsystems is

$$
H_{I}=\left(G_{-} c^{\dagger}+G_{+} a\right) b+\text { H.c. }
$$

( $\hbar=1$ hereafter), where the operators $a, a^{\dagger}$ and $c, c^{\dagger}$ represent cavity 1 and 2 , respectively, and $b, b^{\dagger}$ represent the mechanical mode. $H_{I}$ can be interpreted as a two-step process composed of nondegenerate parametric amplification $[1,35]$ between cavity 1 and the mechanics (term $G_{+} a b+$ H.c.), followed by a beam splitter between the mechanics and cavity 2 (term $G_{-} c^{\dagger} b+$ H.c.), which acts as a means to transfer the amplification to cavity 2 .

Using input-output theory, and assuming $G_{-} \gtrsim G_{+}$, we find the system behaves as a two-port phase-insensitive linear amplifier, as depicted schematically in Fig. 1(c). The output field $a_{\text {out }}$ of cavity 1 has the from

$$
a_{\mathrm{out}}=A_{d} a_{\mathrm{in}}+A_{x} c_{\mathrm{in}}^{\dagger}+F,
$$

and similar for $c_{\text {out }}$ of cavity 2. Here, $A_{d}$ is the direct gain of signals $a_{\text {in }}$ incident on cavity 1 and $A_{x}$ is the cross (frequency-converting) gain of signals incident on cavity 2. Operator $F$ describes the added noise due to the internal modes of the device, viz. $\mathcal{G}^{2} \ll G_{-}^{2}$. In this case, the direct and cross gains are approximately equal, with peak values on resonance 


$$
\left|A_{d}\right|^{2} \approx\left|A_{x}\right|^{2} \approx\left|2 \frac{\kappa_{e}}{\kappa} \frac{4 G_{-}^{2} / \kappa}{\gamma_{\mathrm{eff}}}\right|^{2}
$$

Similar to optomechanical amplifiers powered by a single blue-detuned pump [16], the amplification bandwidth is associated with the effective mechanical linewidth $\gamma_{\text {eff }}$. In the present case, however, the parametric instability threshold is not reached as long as $\gamma_{\text {eff }}>0$ (see Ref. [27]), and further, the bandwidth can be increased beyond the intrinsic linewidth $\gamma$, in principle up to the onset of mode splitting $\gamma_{\mathrm{eff}} \simeq \kappa$ [20]. Moreover, the gain-bandwidth product, $\mathrm{GBW}=\left|A_{d}\right| \gamma_{\mathrm{eff}}$, is determined by $G_{-} \approx G_{+}$, and is not fundamentally limited outside the mode-splitting region. The latter, however, can be fully controlled by $\mathcal{G}$, and, thus, the GBW is rigorously unlimited in the limiting case $G_{-}=G_{+}$.

To characterize the noise performance, we calculate the expected output noise power-spectral density (PSD), $S_{\text {out }}=\frac{1}{2}\left\langle a_{\text {out }}^{\dagger} a_{\text {out }}+a_{\text {out }} a_{\text {out }}^{\dagger}\right\rangle$, from Eq. (2) (and similar for $\left.c_{\text {out }}\right)$. The input-referred added noise is then calculated as $S_{\text {add }}=\left(S_{\text {out }}-S_{\text {in }}\right) /|A|^{2}$, where $A$ is $A_{d}$ or $A_{x}$ for direct and frequency-converting amplification, respectively, and $S_{\text {in }}=1 / 2$ is vacuum input noise driving both cavities. In principle there could also be thermal input noise entering via the ports, but this is typically negligible. In the regime $\gamma \kappa / 4 \ll \mathcal{G}^{2} \ll G_{-}^{2}$ (that is, in the case of high gain and significantly broadened $\gamma_{\text {eff }}$ ), the added noise for direct amplification can be expressed as [31]

$S_{\text {add }} \approx \frac{\gamma \kappa}{4 G_{-}^{2}} \frac{\kappa}{\kappa_{e}}\left(n_{m}^{T}+\frac{1}{2}\right)+\frac{\kappa_{i}}{\kappa_{e}}\left(n_{a}^{T}+n_{c}^{T}+1\right)+\frac{1}{2}$.

Here, the first term originates from the thermal environment of the mechanical oscillator, with an effective temperature $T_{\text {env }}$ corresponding to an occupation $n_{m}^{T} \approx k_{B} T_{\text {env }} / \hbar \omega_{m}$. The second term corresponds to the thermal environment of the cavities, with thermal occupation numbers $n_{a}^{T}$ and $n_{c}^{T}$ for cavity 1 and 2, respectively. Finally, the last term is the vacuum noise of cavity 2 . For large $G_{-}$and $G_{+}$, strong external coupling $\kappa_{e} \gg \kappa_{i}$, and narrow intrinsic mechanical linewidth such that $\gamma \kappa / 4 \ll G_{-}^{2}$, the added noise approaches the quantum limit of one-half quantum. These conditions are available in realizations using either microwave or optical cavities. The fundamental quantum noise is effectively set by cavity 2 (the mode which is not the input). Importantly, the added noise due to the mechanical thermal environment is reduced by a cooperativity-like factor $\gamma \kappa /\left(4 G_{-}^{2}\right)$. Hence, the quantum limit can be nearly reached even when the mechanical oscillator is at a high temperature, in stark contrast to the regular nondegenerate parametric amplifier [35].

Another figure of merit of an amplifier is the capability to handle large signal levels. Josephson parametric amplifiers operating near the quantum limit are poor in this regard. This is because the Josephson energy limits the energy stored in a single junction up to approximately $10^{2}$ photons. Our approach is expected to show a clear improvement because it does not involve nonlinearities close to singlequantum energies. The limiting factor in our system is the critical current of aluminum, which typically allows $10^{8}$ photons inside the cavity. Although Eq. (1) could be realized in Josephson junction systems as well, the large signal handling capabilities favor an electromechanical realization.

In experiment, we measure the performance of our amplifier in a cryogenic environment at a base temperature of $7 \mathrm{mK}$. We inject pumps and signals in both cavities, while measuring the output of cavity 1 . Figure 2(a) shows the direct gain $\left|A_{d}\right|^{2}$, where the signal is injected into cavity 1 . Data are shown as a function of signal frequency for $G_{-}=2 \pi \times$ $355 \mathrm{kHz}$ and several values of $G_{+}$up to $G_{+}=0.99 G_{-}$, corresponding to the highest gain. We achieve a maximum gain of $\left|A_{d}\right|^{2}=41 \mathrm{~dB}$ with a 3-dB bandwidth of $\gamma_{\text {eff }}=1.2 \mathrm{kHz}$, resulting in GBW $=137 \mathrm{kHz}$. The data are in excellent agreement with fits to our model [31]. Figure 2(b) shows an example of frequency-converting
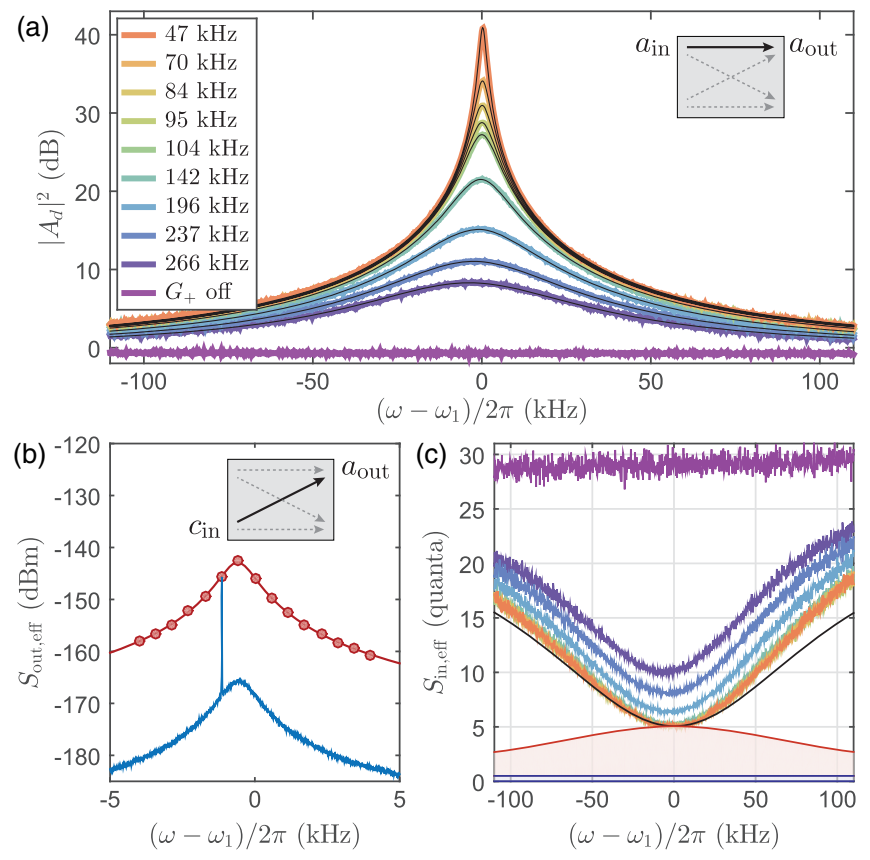

FIG. 2. Two-mode amplifier performance. (a) Direct gain $\left|A_{d}\right|^{2}$ versus signal frequency for fixed $G_{-}$and various values of $G_{+}$ (colored lines, legend shows $\mathcal{G} / 2 \pi$ ) together with theory fits (black lines). Inset: Amplifier configuration; the signal is input to cavity 1. (b) Example output spectrum of cavity 1 (blue line), showing high-gain frequency conversion of a weak signal injected in cavity 2 (narrow peak). The peak height at different frequencies (circles) agrees with the fitted model (red line). Inset: Amplifier configuration. (c) Effective input-referred noise for the same $G_{-}, G_{+}$as in (a) (colored lines). The theory model (black line) is plotted only for the highest gain. The blue shaded area shows the input noise of one-half quanta at each input, and the red shaded area shows the modeled added noise $S_{\text {add }}$. 
amplification. Shown is the output spectrum of cavity 1, while a weak sinusoidal signal is injected into cavity 2 close to resonance, for $G_{-} \approx 2 \pi \times 140 \mathrm{kHz}$ and $\mathcal{G} \approx 2 \pi \times 40 \mathrm{kHz}$. The converted output signal is visible as a narrow peak. The peak output is measured for several frequencies (circles), and the peak gain is $\left|A_{x}\right|^{2} \approx 26 \mathrm{~dB}$.

To accurately quantify the noise performance, we calibrate the total system gain with two independent methods (see also Ref. [31]). First, we compare to a resistor with known 2.9-K thermal noise at the output side of the sample [Fig. 1(a)]. Second, in a subsequent cooldown, we verify the calibration with a tunable noise source at the input side of our sample. Using the latter method, we do not need to know the cable attenuation between the sample and the preamplifier. Using this calibration, we measure the effective noise $S_{\text {out,eff }}$ at the sample output. Figure 2(c) shows the effective input-referred noise spectrum, $S_{\text {in,eff }}=S_{\text {out,eff }} /\left|A_{d}\right|^{2}$, with no signal input, expressed as number of quanta per unit bandwidth and regarding cavity 1 as the input port. $S_{\text {in,eff }}$ is the total system noise: it consists of the input vacuum noise $S_{\text {in }}=1 / 2$ (blue shaded area), the added noise $S_{\text {add }}$ of the mechanical amplifier (red shaded area), as well as the output technical noise $S_{H \text {.eff }}$ added by all further amplification stages [31]. The latter dominates off resonance, but is negligible at high gain. The measured effective noise therefore reduces at increasing gain, saturating at $S_{\text {in,eff }} \simeq 5$ quanta for $\left|A_{d}\right|^{2} \gtrsim 20 \mathrm{~dB}$. This corresponds to an added noise of $S_{\text {add }}=4.6 \pm 1.0$ quanta at the highest gain measurement. The same result applies to frequency-converting amplification (with cavity 2 as the input port), since at high gain $\left|A_{x}\right| \approx\left|A_{d}\right|$. The uncertainty of $S_{\text {add }}$ is dominated by the residual power calibration uncertainty.

In Figs. 3(a)-3(c), we summarize the performance of our amplifier for a wide range of pump powers. For fixed $G_{-}$, the gain-bandwidth product is approximately independent of $\mathcal{G}$, as expected from our model. The highest GBW = $137 \mathrm{kHz}$ as well as the highest absolute gain are achieved at highest $G_{-}$, which is, in turn, limited only by the experimentally available pump power. The optimal bandwidth, while maintaining a total system noise of 5 quanta, is $\gamma_{\text {eff }}=2 \pi \times 11 \mathrm{kHz}$, measured at a gain of $21.5 \mathrm{~dB}$. Figure 3(c) shows the total input-referred noise $S_{\text {in,eff }}$ on resonance, as well as the added noise $S_{\text {add }}$ of our amplifier. At low pump powers, and thus low gain, we observe added noise below 2 quanta, but for high pump powers, $S_{\text {add }}$ increases. Comparing to our theory model, we find that the noise performance is well described by heating of the mechanical environment at high pump powers, up to $n_{m}^{T} \approx$ $5 \times 10^{3}$ quanta. These results are consistent with the heating we observe with standard optomechanical cooling measurements [31]. While heating processes limit the noise performance of the current experiment, they do not pose a fundamental limit on our scheme. The heating could be reduced by increased optomechanical couplings $g_{1}$ and $g_{2}$ and improved mechanical and cavity quality factors, and further depends on the details of device fabrication.
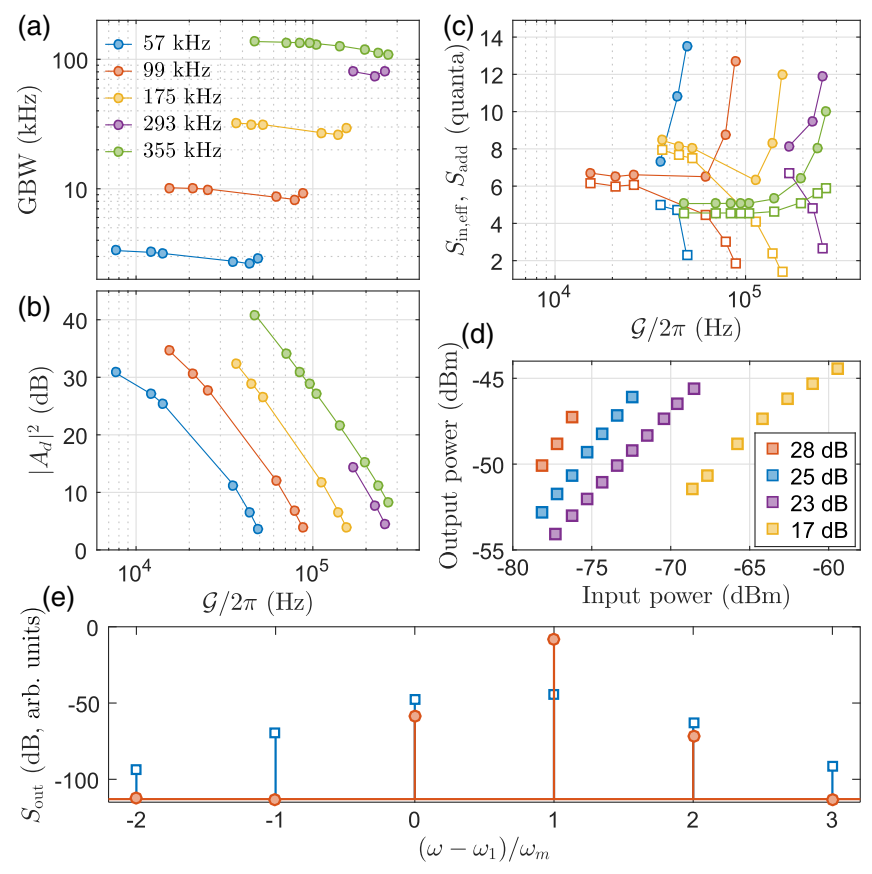

FIG. 3. Amplifier performance and oscillations. (a) Gainbandwidth product for several values of $G_{-}$(legends show $G_{-} / 2 \pi$ ) as a function of $\mathcal{G}$. (b) Peak direct gain and (c) effective input-referred noise (closed circles) and added amplifier noise $S_{\text {add }}$ (open squares) on resonance, for the same parameters as (a). (d) Performance of direct amplification at large input powers, measured for $G_{-} \approx 2 \pi \times 350 \mathrm{kHz}$ and several values of $\mathcal{G}$ (colors). Legend shows the nominal gain for each setting. (e) Harmonic spectra of oscillations in the instability regime without (open squares) and with (closed circles) the red-detuned pump at $\omega_{P-}$ enabled. For the latter case, each harmonic component is measured separately and the horizontal line shows the measurement noise floor.

In addition to operating the pump frequencies at sideband coresonance, our amplifier can be tuned over the cavity linewidth $\kappa$ by shifting the pump frequencies. We measure high-gain $\left(\left|A_{d}\right|^{2}>26 \mathrm{~dB}\right)$ and low-noise $\left(S_{\text {in,eff }} \approx 5\right.$ quanta) amplification over a tuning range of $2 \mathrm{MHz}$. Further tuning could be achieved with tunable microwave cavities [36]. Free-space optical cavities, which have been used in optomechanical systems [15], directly provide tuning over a wide range.

In contrast to existing amplifiers operating near the quantum limit, our amplifier can handle large signal levels. Figure 3(d) shows the output power versus input power, as measured at the sample port, for several nominal gain settings and for the largest input powers where the system remains stable. With a gain of $23 \mathrm{~dB}$ and similar pump powers as in Fig. 2, our amplifier remains stable up to an input power of $-69 \mathrm{dBm}$, or $3 \times 10^{13}$ photons/s. This is $30 \mathrm{~dB}$ higher than reported in Josephson parametric amplifiers [14], and corresponds to a very large dynamic range of $127 \mathrm{~dB}$ in a $1-\mathrm{Hz}$ measurement bandwidth. In these large-signal measurements, the signal-to-noise ratio 
(SNR) is $>100 \mathrm{~dB}$ in a $1-\mathrm{Hz}$ measurement bandwidth, limited by the phase noise of our signal generator. Since we see no evidence of increased noise towards higher input powers, we believe the SNR is equal to the dynamic range, which is 5 orders of magnitude higher than in typical Josephson parametric amplifiers [10], and comparable to the highest values obtained with nearly quantum-limited microwave measurement systems [37].

When increasing $G_{+}$beyond the stability requirement $\gamma_{\text {eff }}>0$, the system undergoes a lasing transition to selfsustained oscillations, which can be a source of spectrally pure electromagnetic radiation [32]. Figure 3(e) shows the measured harmonic spectrum of such oscillations for the two-cavity case (both pumps $\omega_{P+}$ and $\omega_{P_{-}}$on) and for the case of a single pump $\omega_{P+}$. Whereas the single pump case shows many harmonic components, in the twocavity case we observe only a single sideband at $\pm \omega_{m}$ around the pump frequency $\omega_{P+}$. The absence of higher sidebands demonstrates that the mechanical resonator has pure sinusoidal oscillations, which can allow for applications as a source of a clean clock signal.

In a third experiment, we demonstrate coherent frequency conversion of microwave signals without amplification, a frequency-converting analog to an optical beam splitter. This method has been previously demonstrated with optical frequencies [22,23], hybrid microwave-optical systems [29], and very recently in a system similar to ours [30]. Both cavities are pumped at the red mechanical sideband, with pump frequencies $\omega_{P i}=\omega_{i}-\omega_{m}$ for cavity $i=1,2$, respectively. A weak input signal with frequency $\omega_{s}$ is injected into cavity 2 . The converted signal appears at the output of cavity 1 , with a frequency $\omega_{s}^{\prime}=\omega_{s}-\omega_{P 2}+\omega_{P 1}$. The internal conversion efficiency between the two cavities is [22]

$$
\eta_{\text {int }}=\frac{4 G_{1}^{2} G_{2}^{2}}{\left(G_{1}^{2}+G_{2}^{2}+\frac{\gamma \kappa}{4}\right)^{2}},
$$

which approaches unity for $G_{1}^{2}=G_{2}^{2} \gg(\gamma \kappa / 4)$. Here, $G_{i}=\sqrt{n_{i}} g_{i}$. The total conversion efficiency from input to output is $\eta=\eta_{\text {int }} \kappa_{e}^{2} / \kappa^{2}$.

The experimental results are shown in Fig. 4. Figure 4(a) shows the output power spectral density $S\left(\omega^{\prime}\right)$ for several input frequencies close to $\omega_{2}$. The output signal is visible as a narrow peak. The conversion has a bandwidth of $50 \mathrm{kHz}$, corresponding to the mechanical linewidth broadened by optomechanical cooling by both pumps. Figure 4(c) shows the maximum internal conversion efficiency at $\omega_{s}=\omega_{2}$ as a function of output-cavity pump power $P_{P 1}$, for various input-cavity pump powers $P_{P 2}$, together with a global fit of all the data to Eq. (5). We obtain a peak internal efficiency of $\eta_{\text {int }}>0.99$, and the total efficiency is limited only by the cavity coupling $\eta \approx \kappa_{e}^{2} / \kappa^{2} \gtrsim 0.82$. Similar to the two-port amplifier, we observe an increase in noise due to heating of the mechanics at the highest pump powers, resulting in an
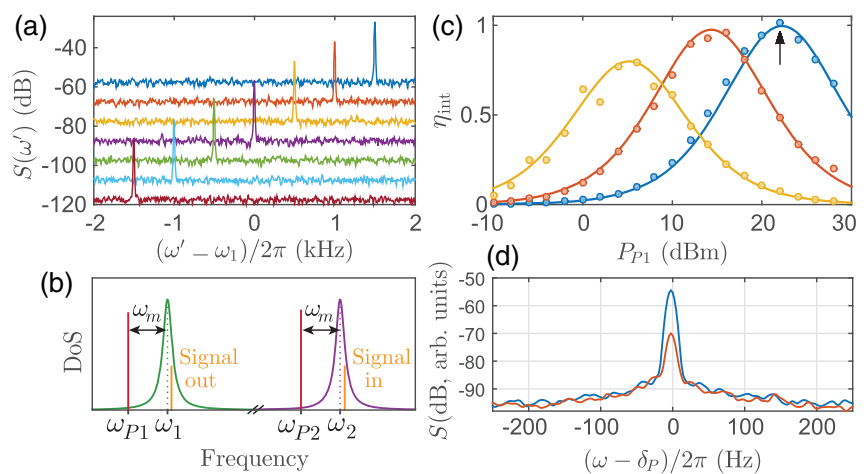

FIG. 4. Frequency conversion without amplification. (a) Output spectrum $S\left(\omega^{\prime}\right)$ (arbitrary units, each curve offset by $10 \mathrm{~dB}$ ) showing the frequency-converted output signal $\omega_{s}^{\prime} \approx \omega_{1}$ for several input frequencies $\omega_{s} \approx \omega_{2}$. (b) Pump and signal configuration. (c) Conversion efficiency on resonance $\left(\omega_{s}=\omega_{2}\right)$ along with fitted theory (see text), for $P_{P 2} \approx 4 \mathrm{dBm}$ (yellow), $14 \mathrm{dBm}$ (red), and $22 \mathrm{dBm}$ (blue), in the same units as $P_{P 1}$. Arrow indicates data shown in (a). (d) Coherence of conversion. Shown are spectra of the mixed-down pump tone $\delta_{P}$ (blue line) and the destructive interference of $\delta_{P}$ and $\delta_{s}$ (red line).

added noise of 3.9 quanta at near-unity conversion efficiency. Using lower pump powers, we obtain an added noise of 1.4 quanta, while retaining a conversion efficiency of $\eta_{\text {int }}=0.95$. In optical and hybrid systems, much lower efficiencies have been reached, mostly limited by $\kappa_{e}$ $[22,23,29]$. To verify coherence of the conversion process, we externally mix the input and output signals, generating their difference frequency $\delta_{s}=\omega_{s}-\omega_{s}^{\prime}$. Similarly, we mix together the two probe tones to generate $\delta_{P}=\omega_{P 2}-\omega_{P 1}$. We then combine $\delta_{s}$ and $\delta_{P}$ on a resistive adder, and with appropriately adjusted relative phase and amplitude we observe $15 \mathrm{~dB}$ of destructive interference between the two tones, as shown in Fig. 4(d). We thus demonstrate coherent frequency conversion near the quantum limit.

Our concept of a two-port optomechanical phaseinsensitive amplifier can be readily applied to other optomechanical systems which have been recently demonstrated, including optical [22,23] and hybrid opticalmicrowave systems [29], providing an essential link to create hybrid networks of otherwise incompatible quantum systems [38-40]. Extending our scheme to multiple cavity modes creates a universal hub for electromechanical or optomechanical signals at the quantum level [41,42], with high-gain, high-power amplification enabling interconnection of remote systems. At optical frequencies [43], the quantum limit of added noise should be accessible at room temperature. Given that in the present microwave experiment the added noise is limited by residual heating of the mechanical resonator, we expect the quantum limit can be reached by, first of all, improving the coupling efficiency. With a factor of 2 higher coupling, and an order of magnitude higher pump powers feasible, in particular, in 3D cavities [44] with niobium technology, the device can 
operate at MHz-range bandwidth close to the standard quantum limit at $4-\mathrm{K}$ temperatures, hence presenting an attractive alternative to HEMT amplifiers in narrow-band microwave measurements.

We thank Visa Vesterinen and Pasi Lähteenmäki for useful discussions. This work was supported by the Academy of Finland (Contract No. 250280, CoE LTQ, 275245) and by the European Research Council (240387NEMSQED, 240362-Heattronics, 615755-CAVITYQPD). The work benefited from the facilities at the Micronova Nanofabrication Center and at the Low Temperature Laboratory infrastructure.

C. F. O.-K. carried out the practical work, analyzed data, and wrote the paper. E. D. and J.-M. P. developed the device fabrication process. T. T. H. and F. M. developed the theory. M. A. S initiated and supervised the project.

[1] A. A. Clerk, M. H. Devoret, S. M. Girvin, F. Marquardt, and R. J. Schoelkopf, Introduction to Quantum Noise, Measurement, and Amplification, Rev. Mod. Phys. 82, 1155 (2010).

[2] R. Vijay, D. H. Slichter, and I. Siddiqi, Observation of Quantum Jumps in a Superconducting Artificial Atom, Phys. Rev. Lett. 106, 110502 (2011).

[3] J. D. Teufel, T. Donner, D. Li, J. W. Harlow, M. S. Allman, K. Cicak, A. J. Sirois, J. D. Whittaker, K. W. Lehnert, and R. W. Simmonds, Sideband Cooling of Micromechanical Motion to the Quantum Ground State, Nature (London) 475, 359 (2011).

[4] L. Steffen, Y. Salathe, M. Oppliger, P. Kurpiers, M. Baur, C. Lang, C. Eichler, G. Puebla-Hellmann, A. Fedorov, and A. Wallraff, Deterministic Quantum Teleportation with FeedForward in a Solid State System, Nature (London) 500, 319 (2013).

[5] R. Vijay, C. Macklin, D. H. Slichter, S. J. Weber, K. W. Murch, R. Naik, A. N. Korotkov, and I. Siddiqi, Stabilizing Rabi Oscillations in a Superconducting Qubit Using Quantum Feedback, Nature (London) 490, 77 (2012).

[6] J. Kelly et al., State Preservation by Repetitive Error Detection in a Superconducting Quantum Circuit, Nature (London) 519, 66 (2015).

[7] D. J. Wilson, V. Sudhir, N. Piro, R. Schilling, A. Ghadimi, and T. J. Kippenberg, Measurement-Based Control of a Mechanical Oscillator at Its Thermal Decoherence Rate, Nature (London) 524, 325 (2015).

[8] C. M. Caves, Quantum Limits on Noise in Linear Amplifiers, Phys. Rev. D 26, 1817 (1982).

[9] B. Yurke, L. R. Corruccini, P. G. Kaminsky, L. W. Rupp, A. D. Smith, A.H. Silver, R.W. Simon, and E. A. Whittaker, Observation of Parametric Amplification and Deamplification in a Josephson Parametric Amplifier, Phys. Rev. A 39, 2519 (1989).

[10] M. A. Castellanos-Beltran, K. D. Irwin, G. C. Hilton, L. R. Vale, and K. W. Lehnert, Amplification and Squeezing of Quantum Noise with a Tunable Josephson Metamaterial, Nat. Phys. 4, 929 (2008).
[11] N. Bergeal, F. Schackert, M. Metcalfe, R. Vijay, V.E. Manucharyan, L. Frunzio, D. E. Prober, R. J. Schoelkopf, S. M. Girvin, and M. H. Devoret, PhasePreserving Amplification Near the Quantum Limit with a Josephson Ring Modulator, Nature (London) 465, 64 (2010).

[12] P. Lähteenmäki, V. Vesterinen, J. Hassel, H. Seppä, and P. Hakonen, Josephson Junction Microwave Amplifier in SelfOrganized Noise Compression Mode, Sci. Rep. 2, 276 (2012).

[13] C. Eichler, Y. Salathe, J. Mlynek, S. Schmidt, and A. Wallraff, Quantum-Limited Amplification and Entanglement in Coupled Nonlinear Resonators, Phys. Rev. Lett. 113, 110502 (2014).

[14] C. Macklin, K. O’Brien, D. Hover, M. E. Schwartz, V. Bolkhovsky, X. Zhang, W. D. Oliver, and I. Siddiqi, A NearQuantum-Limited Josephson Traveling-Wave Parametric Amplifier, Science 350, 307 (2015).

[15] M. Aspelmeyer, T. J. Kippenberg, and F. Marquardt, Cavity Optomechanics, Rev. Mod. Phys. 86, 1391 (2014).

[16] F. Massel, T. T. Heikkilä, J.-M. Pirkkalainen, S. U. Cho, H. Saloniemi, P. J. Hakonen, and M. A. Sillanpää, Microwave Amplification with Nanomechanical Resonators, Nature (London) 480, 351 (2011).

[17] T. G. McRae and W.P. Bowen, Near Threshold AllOptical Backaction Amplifier, Appl. Phys. Lett. 100, 201101 (2012).

[18] H. Li, Y. Chen, J. Noh, S. Tadesse, and M. Li, Multichannel Cavity Optomechanics for All-Optical Amplification of Radio Frequency Signals, Nat. Commun. 3, 1091 (2012).

[19] T. Bagci, A. Simonsen, S. Schmid, L. G. Villanueva, E. Zeuthen, J. Appel, J. M. Taylor, A. Sorensen, K. Usami, A. Schliesser, and E. S. Polzik, Optical Detection of Radio Waves through a Nanomechanical Transducer, Nature (London) 507, 81 (2014).

[20] A. Metelmann and A. A. Clerk, Quantum-Limited Amplification via Reservoir Engineering, Phys. Rev. Lett. 112, 133904 (2014).

[21] I. S. Grudinin, H. Lee, O. Painter, and K. J. Vahala, Phonon Laser Action in a Tunable Two-Level System, Phys. Rev. Lett. 104, 083901 (2010).

[22] C. Dong, V. Fiore, M. C. Kuzyk, and H. Wang, Optomechanical Dark Mode, Science 338, 1609 (2012).

[23] J. T. Hill, A. H. Safavi-Naeini, J. Chan, and O. Painter, Coherent Optical Wavelength Conversion via Cavity Optomechanics, Nat. Commun. 3, 1196 (2012).

[24] J. F. Poyatos, J. I. Cirac, and P. Zoller, Quantum Reservoir Engineering with Laser Cooled Trapped Ions, Phys. Rev. Lett. 77, 4728 (1996).

[25] K. W. Murch, U. Vool, D. Zhou, S. J. Weber, S. M. Girvin, and I. Siddiqi, Cavity-Assisted Quantum Bath Engineering, Phys. Rev. Lett. 109, 183602 (2012).

[26] S. Shankar, M. Hatridge, Z. Leghtas, K. M. Sliwa, A. Narla, U. Vool, S. M. Girvin, L. Frunzio, M. Mirrahimi, and M. H. Devoret, Autonomously Stabilized Entanglement between Two Superconducting Quantum Bits, Nature (London) 504, 419 (2013).

[27] Y.-D. Wang and A. A. Clerk, Reservoir-Engineered Entanglement in Optomechanical Systems, Phys. Rev. Lett. 110, 253601 (2013). 
[28] C. A. Regal, J. D. Teufel, and K. W. Lehnert, Measuring Nanomechanical Motion with a Microwave Cavity Interferometer, Nat. Phys. 4, 555 (2008).

[29] R. W. Andrews, R. W. Peterson, T. P. Purdy, K. Cicak, R. W. Simmonds, C. A. Regal, and K. W. Lehnert, Bidirectional and Efficient Conversion between Microwave and Optical Light, Nat. Phys. 10, 321 (2014).

[30] F. Lecocq, J. B. Clark, R. W. Simmonds, J. Aumentado, and J. D. Teufel, Mechanically Mediated Microwave Frequency Conversion in the Quantum Regime, Phys. Rev. Lett. 116, 043601 (2016).

[31] See Supplemental Material at http://link.aps.org/ supplemental/10.1103/PhysRevX.6.041024 for details on the theoretical model, experimental setup, and data analysis.

[32] A. Nunnenkamp, V. Sudhir, A. K. Feofanov, A. Roulet, and T. J. Kippenberg, Quantum-Limited Amplification and Parametric Instability in the Reversed Dissipation Regime of Cavity Optomechanics, Phys. Rev. Lett. 113, 023604 (2014).

[33] A. Metelmann and A. A. Clerk, Nonreciprocal Photon Transmission and Amplification via Reservoir Engineering, Phys. Rev. X 5, 021025 (2015).

[34] L. D. Tóth, N. R. Bernier, A. Nunnenkamp, E. Glushkov, A. K. Feofanov, and T. J. Kippenberg, Engineered Dissipative Reservoir for Microwave Light Using Circuit Optomechanics, arXiv:1602.05180.

[35] W. H. Louisell, A. Yariv, and A. E. Siegman, Quantum Fluctuations and Noise in Parametric Processes. I., Phys. Rev. 124, 1646 (1961).

[36] R. W. Andrews, A. P. Reed, K. Cicak, J. D. Teufel, and K. W. Lehnert, Quantum-Enabled Temporal and Spectral
Mode Conversion of Microwave Signals, Nat. Commun. 6, 10021 (2015).

[37] B. H. Eom, P. K. Day, H. G. LeDuc, and J. Zmuidzinas, A Wideband, Low-Noise Superconducting Amplifier with High Dynamic Range, Nat. Phys. 8, 623 (2012).

[38] Z.-L. Xiang, S. Ashhab, J. Q. You, and F. Nori, Hybrid Quantum Circuits: Superconducting Circuits Interacting with Other Quantum Systems, Rev. Mod. Phys. 85, 623 (2013).

[39] J. Bochmann, A. Vainsencher, D. D. Awschalom, and A. N. Cleland, Nanomechanical Coupling between Microwave and Optical Photons, Nat. Phys. 9, 712 (2013).

[40] G. Kurizki, P. Bertet, Y. Kubo, K. Mølmer, D. Petrosyan, P. Rabl, and J. Schmiedmayer, Quantum Technologies with Hybrid Systems, Proc. Natl. Acad. Sci. U.S.A. 112, 3866 (2015).

[41] K. Stannigel, P. Komar, S. J. M. Habraken, S. D. Bennett, M. D. Lukin, P. Zoller, and P. Rabl, Optomechanical Quantum Information Processing with Photons and Phonons, Phys. Rev. Lett. 109, 013603 (2012).

[42] M. Schmidt, M. Ludwig, and F. Marquardt, Optomechanical Circuits for Nanomechanical Continuous Variable Quantum State Processing, New J. Phys. 14, 125005 (2012).

[43] M. Eichenfield, J. Chan, R. M. Camacho, K. J. Vahala, and O. Painter, Optomechanical Crystals, Nature (London) 462 , 78 (2009).

[44] M. Yuan, V. Singh, Y. M. Blanter, and G. A. Steele, "Large Cooperativity and Microkelvin Cooling with a Three-Dimensional Optomechanical Cavity, Nat. Commun. 6, 8491 (2015). 